\title{
Fetal ECG Extraction for Fetal Monitoring Using SWRLS Adaptive Filter
}

\author{
P. Sunita ${ }^{1}$, G. Prathibha ${ }^{2}$ \\ ${ }^{1}$ Student, Electronics and Communication Engineering, Acharya Nagarjuna University, Guntur, India \\ ${ }^{2}$ Assistant.Professor, Electronics and Communication Engineering, Acharya Nagarjuna University, Guntur, India
}

\begin{abstract}
Fetal electrocardiogram (Fetal ECG) extraction is an interesting as well as a difficult problem in signal processing. The original FECG signal is nevertheless very complex and severely contaminated by external disturbances or noises. Identification of these cases during early pregnancy reduces risks by timely treatment or planned delivery. This paper presents simulation results of fetal ECG signal after removing noise by sliding window RLS adaptive filter. From this obtained fetal ECG signal we can find the abnormalities by $R-R$ peak detection and care will be taken by the respective physician.
\end{abstract}

Keywords: Fetal ECG, Maternal ECG, Adaptive filter, Recursive least square algorithm, Sliding window RLS algorithm

\section{Introduction}

Fetal heart rate (FHR) monitoring is one of the possible solutions to test fetal well-being and to diagnose possible abnormalities. Fetal monitoring during pregnancy stage enables the physician to diagnose and recognize the pathologic condition. The electrocardiogram (ECG) is the simplest non invasive diagnostic method for various diseases. Fetal ECG (FECG) signal reflects the electrical activity of the fetal heart and provides valuable information of its physiological state. Fetal ECG signal can be extracted from maternal ECG by many algorithms of adaptive filter. Noor K.Muhsin proposed Noise removal of ECF signal using recursive least algorithm [1].Roshny Jose George proposed Design of an adaptive filtering algorithm for noise cancellation [2].This paper shows extraction of FECG signal for fetal monitoring by using sliding window RLS adaptive filter.

\section{Adaptive Filter}

Adaptive filters are variable filters whose filter coefficients are adjustable or modifiable automatically to improve its performance in accordance with some criterion, allowing the filter to adapt the changes in the input signal characteristic. Because of their self-adjusting performance and in-built flexibility, adaptive filters have found use in many diverse applications such as echo cancelling, radar signal processing, and noise cancelling and biomedical signal enhancement. It is non-linear and time variant and best suits when signal conditions are slowly changing. The basic operation of adaptive filter involves two processes. They are:

i) Filtering process, which produces an output signal in response to a given input signal.

ii) Adaptation process, which aims to adjust the filter parameters to the environment.

\section{Adaptive Noise Canceller}

This method is used for non-stationary type noise or interference which is not necessarily a random process. Adaptive filters are systems with a linear filter that uses a transfer function which is controlled by different variable parameters and these parameters can be adjusted according to an optimization algorithm. Most adaptive filters re adjustable digital filters owing to the complexity of the optimization algorithms used. In case of an ANC no information about the signal and noise characteristic is available and both the noise and signal are uncorrelated. Another reference signal is obtained from a second source which is strongly correlated with the noise but uncorrelated with the signal, When the signal and noise are stationary, an adaptive filter acts as a fixed filter whereas it acts as a notch filter or comb filter for a periodic interference. Least Mean Squares (LMS), Recursive Least Squares (RLS) filters are a few examples of adaptive filters and all these filters described above can only be applicable for additive noise.

\section{Methodology}

The electrocardiogram (ECG) signal for both the mother and fetus are retrieved from the given data that is sampled at $500 \mathrm{~Hz}$ using a smoothing filter to give the discrete data a somewhat smoother shape. The filter used here is a digital filter known as the Savitzy-Galoy Filter. The main purpose of using this filter is to smooth the signal by increasing the signal-to-noise ratio (SNR) without distorting the signal significantly. This filter uses a convolution method to achieve the goal. The heart rate of a fetus is significantly higher than that of the maternal heart rate which is in this case approximately a rate of 85 beats per minute whereas for the fetus $t$ is approximately 132 beats per minute. In general, the fetus heart bets faster than that o the mother, with a range of rates from 120 to 160 beats per minute. The fetal electrocardiogram signal has much weaker amplitude as compared to its maternal counterpart. For example here the mother ECG signal has a peak of just 3.5 milli volts whereas the fetal ECG signal has a peak of just 0.25 millivolts.ECG signals are taken from two different locations of the mother's body, the chest and the abdomen. The chest signal gives the original mother ECG signal whereas the signal obtained from the abdomen is a mixture of both mother and fetus heartbeat signals usually dominated by the maternal component propagated from the chest cavity to the abdomen. A linear FIR filter can be used with 10 randomized coefficients in order to describe this path. There 


\section{International Journal of Science and Research (IJSR) \\ ISSN (Online): 2319-7064 \\ Index Copernicus Value (2013): 6.14 | Impact Factor (2015): 6.391}

may be some additional broadband interference associated with both mother and fetus signals that can be eliminated by the addition of a small amount of uncorrelated Gaussian noise. The task performed by the adaptive filter is to adaptively remove maternal component from the fetus heartbeat signal. To do so it needs a reference signal which is nothing but the signal generated from the maternal ECG itself. Like the fetal ECG signal the maternal ECG signal is also expected to contain some additive broadband noise.

\section{Algorithm Used For Extraction}

The extraction method described here is based on a MATLAB code that consists of 4 simple steps as described in the flowchart of the proposed algorithm shown in the fig. 1

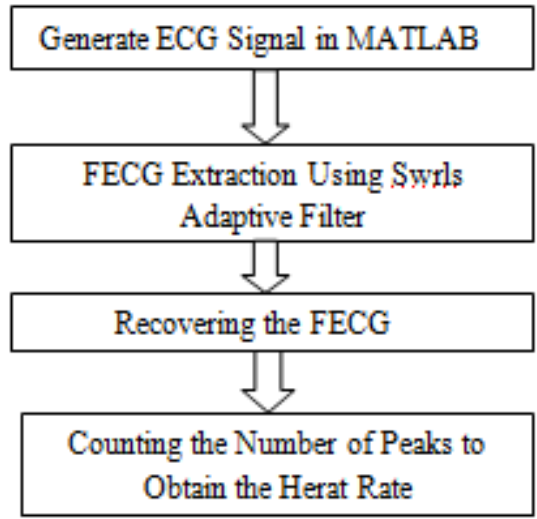

Figure 1: Algorithm used for extraction of fetal ECG

The different steps proposed in the algorithm are briefly described below,

\section{Generation of the ECG signals using MATLAB}

MATLAB is an easy-to use tool which is very helpful in the extraction of the fetal ECG (FECG) signal from the abdominal ECG AECG).Using MATLAB we generate the signal on which the task can be performed and implemented easily. MATLAB contains a function Savitzy-Galoy filter function and using this command the required signals are generated. MATLAB coding is used to simulate the shapes of the ECG signals for both the mother and the baby. The peak amplitude of this maternal signal is 3.5 mill volts and the heart rate being 85 beats per minute. But the fetus heart bats noticeably faster than that of the mother's ranging between 120 to 160 beats per minute as stated previously.

\section{FECG Signal extraction Methods}

\section{a) Adaptive noise canceller (ANC)}

Two signals are needed to remove background articrafts including different noises or interference already present in the FECG signal by using adaptive filtering. The first one is the FECG signal added with MECG signal and the second being the reference signal which is cancelled to get the FECG signal. The reference signal is nothing but equal to the MECG signal itself. The different noise present in both the signals must be well correlated i.e., the noise in the primary and secondary signals. In the fetal ECG extraction procedure adaptive filters are used to adaptively remove a mother's heartbeat signal from the fetal ECG signal to get the heartbeat signal of the baby or the fetus. When the operating environment is stationary, these filters possess constant shape and orientation for the error-performance surface. But for operation in an environment that is not stationery, the bottom of this surface moves continually along with the chances of changing orientation and curvature. Hence, for non-stationery inputs, the filter seeks the bottom of the error performance surface along with continually tracking it down. The block diagram for a basic ANC system is shown below

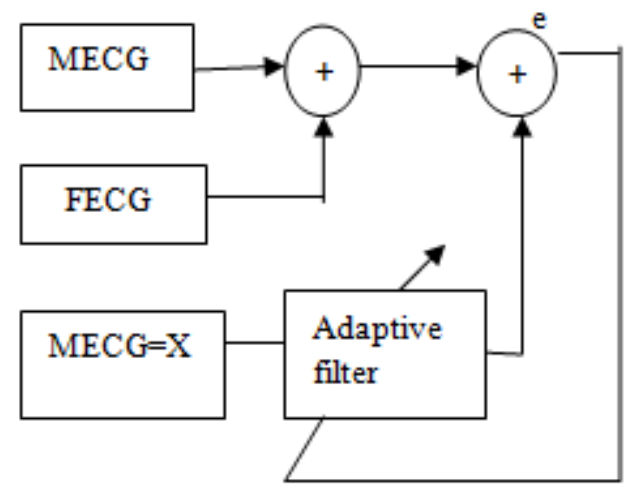

Figure 2: Adaptive noise cancellation systems

\section{b) High Frequency Removal Using Digital Filter}

FIR and IIR filters are the two primary types of filters widely used in digital signal processing (DSP).among those FIR filters can be easily designed in linear phase and some of the calculations can be omitted b using FIR filters, thus providing some important computational efficiency. In order to count the fetal heart rate the R-peaks of the FECG signal must be extracted. A Fir filter with appropriate filter coefficients is used to remove high frequencies in order to achieve this goal. Then a threshold value is set and the peaks with higher values than the threshold are detected as the Rpeaks of the FECG signal. The number of R-peaks is counted and from the R-r interval the fetal hart rate is calculated.

\section{SWRLS Algorithm}

SWRLS is nothing but sliding window recursive least square algorithm. SWRLS has better tracking ability and easy to work with noisy system and is mainly used to increase the convergence speed i.e.it has superior trade off among tracking, robustness and convergence speed when compared to remaining algorithms of adaptive filter. It has good estimation in different SNR which produces less noise when compared to different algorithms of adaptive filter.

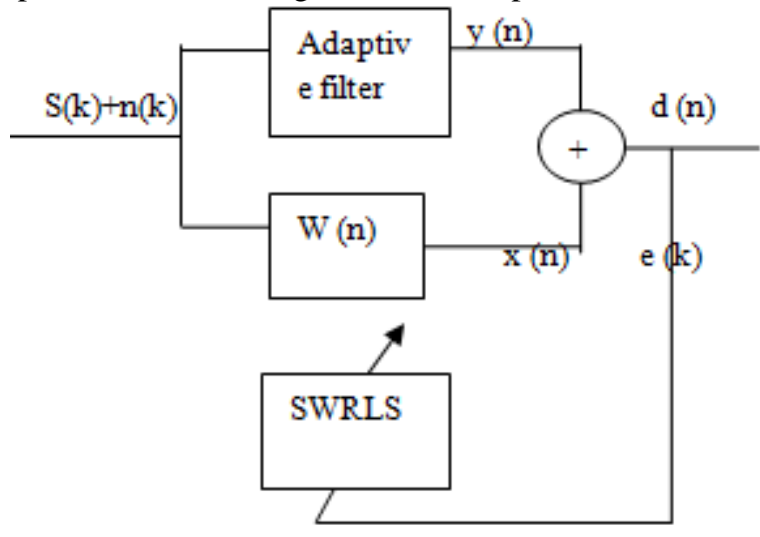

Figure 3: General structure of adaptive SWRLS filter 


\section{International Journal of Science and Research (IJSR) \\ ISSN (Online): 2319-7064 \\ Index Copernicus Value (2013): 6.14 | Impact Factor (2015): 6.391}

In fig 3,input signal i.e. $s(\mathrm{k})+\mathrm{n}(\mathrm{k})$ which contains noise $\mathrm{n}(\mathrm{k})$ is given to the adaptive filter and error is removed by using SWRLS filter and thus desired signal $d(n)$ is obtained from which abnormalities can be detected and particular care will be taken by the respective physician.

\section{Results \& Discussions}

The signal shown in fig4 is the maternal ECG signal which contains both noise and fetal ECG signals. Heart beat rate of maternal signal is 85 beats per minute.

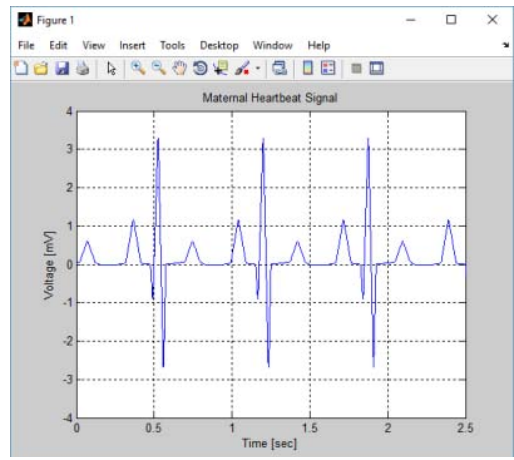

Figure 4: Maternal heart beat signal

The signal shown in fig 5 is the fetal ECG signal which is obtained after removing noise. Fetal ECG has heart rate of $120-160$ beats per minute which is higher than that of Maternal ECG signal.

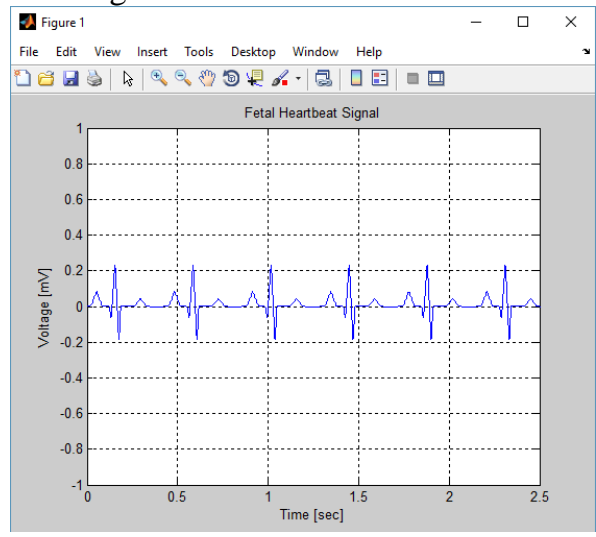

Figure 5: Fetal ECG signal

The signal shown in fig 6 is the noise signal which is obtained by sliding window RLS filter.

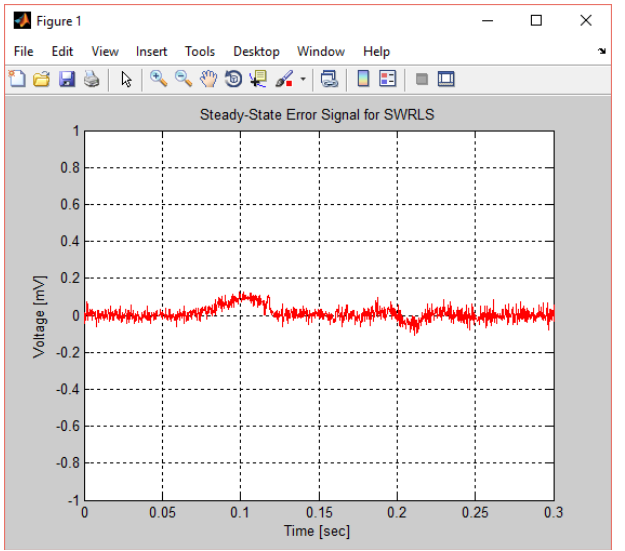

Figure 6: Removal of error signal by SWRLS

\section{Performance Comparison of Adaptive Filter}

The performance metrics for various adaptive filters is measured according to the measuring factors like signal to noise ratio (SNR), computational speed, RMS, distortion which is shown in below table 1 .

Signal to noise ratio (SNR) is given as the ratio of signal power to the noise power and SNR should be high for the better performance of the signal. Distortion is the alteration of the original shape (or other characteristic) of something such as an object, image, sound or waveform and it should be minimum for any signal.

Table 1: Comparison of adaptive filters

\begin{tabular}{|c|c|c|c|c|c|}
\hline $\begin{array}{c}\text { S. } \\
\text { No }\end{array}$ & Algorithm & SNR & $\begin{array}{c}\text { Computational } \\
\text { speed }\end{array}$ & RMS & Distortion \\
\hline 1 & LMS & 14.3 & 1.532 & 0.165 & -15.607 \\
\hline 2 & RLS & 24.2 & 3.388 & 0.053 & -25.48 \\
\hline 3 & SWRLS & 43.73 & 5.718 & 165.23 & -44.3623 \\
\hline
\end{tabular}

As signal to noise ratio is high and distortion is less for SWRLS, it is the best technique to extract the fetal ECG signal and to detect the abnormalities in the signal.

\section{Conclusion}

Thus fetal ECG signal is extracted from maternal signal and noise is removed by using sliding window RLS adaptive filter and abnormalities can be detected by counting R-R peaks of fetal ECG signal. If the count is not equal to desired number then it indicates that abnormalities are present and particular care will be taken by the respective physician.

\section{References}

[1] G.V.P.Chandra sekhar yadav \&B.Ananda Kumar, Study of different adaptive algorithms for noise cancellation in real time environment, International journal of computer applications,,volumw96-no-10,jum=ne 2014

[2] Jyoti Dhiman.Shadeb Ahmad, Kuldeep Gulia, Comparison between adaptive filter algorithms, IJSETR volume 2, issue 5, may 2013.

[3] Noor K.Muhsin, Noise removal of ECG signal using recursive least square algorithm, Al-Khwarizmi engineering journal,vol.7,no.1,pp 12-21(2011).

[4] Frantisek dohnal, Generalized frequency domain LMS adaptive filter, Radio engineering, vol.4, no.2, June 1995

[5] Ricardo Merchand, A unified approach to structured covariances: fast generalized sliding window RLS recursion for arbitrary basis, IEEE transactions on signal processing, vol.61, no, 23, december1, 2013

[6] Steven van varenbergh, Javier via and Igancio santmaria, Nonlinear system identification using a new sliding window kernel RLS algorithm, Journal of communication, vol.2, no.3, May 2007.

\section{Author Profile}

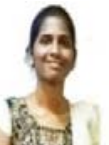

P. Sunita studying final year B-Tech in Electronics and Communication Engineering at Acharya Nagarjuna University College of Engineering and Technology, Guntur, India. Her areas of interest are 


\section{International Journal of Science and Research (IJSR) \\ ISSN (Online): 2319-7064}

Index Copernicus Value (2013): 6.14 | Impact Factor (2015): 6.391

Digital and signal processing.

G. Prathibha currently working as an assistant professor in Electronics and Communication Engineering at Acharya Nagarjuna University College of Engineering and Technology, Guntur, India. Her areas of interests are Image processing, Signal processing and Recognition of pattern diagrams. 